\title{
Wind Profile Prediction Using Linear Markov Chains: A Linear Algebra Approach
}

\author{
M. I. Garcia and T. Gongadze
}

\begin{abstract}
To predict the future wind speed and wind direction is of relevance to the wind industry to maximize the power generation. In this regards, this article describes a methodology for the construction of predictive models based on linear Markov chains under linear algebra point of view. The model analyzes the direction and speed of the wind obtained from a meteorological station. This Model allows making a precise study of wind direction and speeding data; figure out the stability, the most common direction or speed, its behaviour depending on the hours or seasons.
\end{abstract}

Keywords- MATLAB, Markov Chains, eigenvectors, eigenvalues, stationary distribution.

\section{INTRODUCCIÓN}

工 A energía eólica forma parte de las energías llamadas renovables cuya fuente es la fuerza del viento. La manera más habitual de aprovechar esta energía es mediante la utilización de aerogeneradores o turbinas de viento. Un factor condicionante para la operatividad y mantenimiento de los parques eólicos son las condiciones climáticas que por lo general se caracterizan por la dirección del viento y por la velocidad del viento. Por lo tanto, en el momento de decidir estrategias óptimas de operatividad y mantenimiento de aerogeneradores se deben tener en cuenta dichas condiciones climáticas, [10].

El objetivo de este estudio es crear un modelo de análisis de la dirección y velocidad del viento centrado en proporcionar un marco teórico para el análisis de la variabilidad de la velocidad y dirección del viento. El método que se propone para realizar dicho estudio es mediante cadenas de Markov lineales en tiempo discreto tratadas desde el punto de vista de Álgebra lineal. Dichas cadenas suelen ser comúnmente utilizadas en áreas en las que es preciso modelar el comportamiento de ciertos sistemas tanto de ingeniería, economía como de medicina entre otros, bajo condiciones de incertidumbre a través del tiempo [2], [5], [6].

El modelo propuesto se ha aplicado a la obtención de mtrices de Markov proveniente de datos de viento reales provenientes de la estación meteorológica de "Trinidad" de Iturgoyen cuya localización geográfica es $42^{\circ} 46^{\prime} 34^{\prime \prime} \mathrm{N}$, $1^{\circ} 57^{\prime} 15^{\prime \prime} \mathrm{O},[7]$.

\section{NOMENCLATURA}

- R: Conjunto de números reales

- $\mathrm{M}_{\mathrm{nxm}}(\mathbf{R})$ : Conjunto de matrices con $n$ filas y $m$ columnas.

M. I. García, Universitat Politècnica de Catalunya, Barcelona, España. maria.isabel.garcia@upc.edu

T. Gongadze, Universitat Politècnica de Catalunya, Barcelona, España tornike.gongadze@gmail.com

\section{CADENAS DE MARKOV}

Las cadenas de Markov [1], [3], [8], son modelos matemáticos estructurados basados en el álgebra lineal. Estas constituyen una buena herramienta para el estudio del comportamiento de procesos que evolucionan de forma no determinista, a lo largo del tiempo sobre un conjunto de estados posibles. Esta herramienta es considerada esencial en disciplinas como la ingeniería, la economía y la investigación operativa entre otros, en cada caso se utiliza dicha herramienta para describir experimentos que se realizan varias veces de la misma manera y en el que el resultado de cada uno de los ensayos depende únicamente del ensayo inmediatamente anterior.

De manera más formal, una cadena de Markov es un proceso aleatorio sin memoria; a los posibles valores que puede tomar la variable aleatoria reciben el nombre de estados. Esto es, un sistema que evoluciona en el tiempo $(\mathrm{k}=$ $0,1, \ldots)$, una variable $X$ está en el estado $i_{\mathrm{k}}$ en el instante $\mathrm{k}$, si en el instante $\mathrm{k}-1$ está en el estado $\mathrm{i}_{\mathrm{k}-1}$ :

$$
\begin{gathered}
P\left(X_{k}=i_{k} \mid X_{0}=i_{0}, \ldots \ldots, X_{k-1}=i_{k-1}\right)= \\
P\left(X_{k}=i_{k} \mid X_{k-1}=i_{k-1}\right)=p_{i_{k-1}, i_{k}}
\end{gathered}
$$

Intuitivamente, esta ecuación implica que, conocido el estado "presente" de un sistema, el estado "futuro" es independiente de su estado "pasado".

Las cadenas de Markov se pueden describir matricialmente de la siguiente manera:

Si $p_{1}(k), \ldots, p_{\mathrm{n}}(k)$ son las probabilidades de los $\mathrm{n}$ estados en el tiempo $k$ y $p_{i j}(k)$ la probabilidad de la evolución del estado i al $\mathrm{j}$ en el tiempo $k, 1 \leq \mathrm{i}, \mathrm{j} \leq \mathrm{n}$.

$\left(\begin{array}{c}p_{1}(k+1) \\ \vdots \\ p_{n}(k+1)\end{array}\right)=\left(\begin{array}{ccc}p_{11}(k) & \cdots & p_{1 n}(k) \\ \vdots & \ddots & \vdots \\ p_{n 1}(k) & \cdots & p_{n n}(k)\end{array}\right)\left(\begin{array}{c}p_{1}(k) \\ \vdots \\ p_{n}(k)\end{array}\right)$

La matriz

$$
A=\left(\begin{array}{ccc}
p_{11}(k) & \cdots & p_{1 n}(k) \\
\vdots & \ddots & \vdots \\
p_{n 1}(k) & \cdots & p_{n n}(k)
\end{array}\right)
$$

recibe el nombre de matriz de transición de estados.

Si la matriz $A$ no depende de k, se dice que la cadena de Markov es invariante en el tiempo, en cuyo caso se cumple que: 


$$
\left(\begin{array}{c}
p_{1}(k+1) \\
\vdots \\
p_{n}(k+1)
\end{array}\right)=\left(\begin{array}{ccc}
p_{11} & \cdots & p_{1 n} \\
\vdots & \ddots & \vdots \\
p_{n 1} & \cdots & p_{n n}
\end{array}\right)^{k}\left(\begin{array}{c}
p_{1}(0) \\
\vdots \\
p_{n}(0)
\end{array}\right)
$$

Puesto que uno de los objetivos que se persiguen al modelar una determinada situación es el de poder conocer su comportamiento a largo plazo y teniendo en cuenta (1), la matriz de transición de estados $A$ nos resuelve el problema de encontrar el término general de la sucesión de estados. El límite en caso de que exista, de esta sucesión cuando el tiempo tiende a infinito, nos proporciona un método para estudiar el comportamiento a largo plazo de la cadena de Markov.

Para obtener este límite, recurrimos al álgebra lineal.

Genéricamente la matriz $A$ admite una base de vectores propios $v_{1,}, \ldots, v_{n}$, esto es tal que $A\left(v_{i}\right)=\lambda_{i} v_{i} \quad$ (los valores $\lambda_{i}$ reciben el nombre de valores propios asociados) por lo que, fijada una condición inicial, esta se puede expresar como combinación lineal única de esta base:

$$
p(0)=\mathrm{a}_{1} v_{i}+\cdots+\mathrm{a}_{n} v_{n}
$$

Por lo que y en virtud de la linealidad, se tiene:

$$
p(k)=\lambda_{1}{ }^{k} \mathrm{a}_{1} v_{i}+\cdots,+\lambda_{n}{ }^{k} \mathrm{a}_{n} v_{n}
$$

Con esta expresión de $p(k)$ es fácil estudiar la evolución a lo largo del tiempo de la cadena de Markov, es decir, es fácil analizar si existe un punto de equilibrio estable, es decir si existe una solución constante $\pi$ de manera que todas las demás soluciones tiendan a esta:

$$
\lim p(k)=\pi
$$

Al punto de equilibrio lo denominaremos distribución estacionaria de la cadena.

En el caso más general la matriz $A$ reduce a su forma triangular de Jordan, forma en la que también es fácil deducir el valor de $p(k)$ en función de los valores y vectores propios generalizados de la matriz, (ver [3] para más detalles).

La existencia de la distribución estacionaria en el caso de las cadenas de Markov lineales puede ser estudiada a partir del siguiente teorema:

Teorema (Teorema de Frobenius). Sea $A=\left(\mathrm{p}_{\mathrm{ij}}\right)$ la matriz de transición de un proceso modelado como cadena de Markov de orden $n$, irreducible. Se tiene que:

i) $\quad \kappa=1$ es valor propio de $A$.

ii) Cualquier valor propio u de $A$ verifica $\mathrm{v} \leq 1$.

iii) Existe un vector propio $\pi$ de valor propio 1, cuyas coordenadas son todas mayores o iguales que cero. Sin pérdida de generalidad, podemos asumir que la suma de sus coordenadas es 1 .

Esto nos lleva a afirmar que hay una cierta evidencia de la existencia de un estado estable, por lo tanto, las probabilidades de que cada vez que avance el tiempo, los estados van a tender a converger hacia un mismo estado (el correspondiente a la distribución estacionaria).

La distribución estacionaria puede ser computada utilizando métodos iterativos (ver por ejemplo [9]), sin embargo aquí, tal y como hemos indicado, vamos a usar técnicas de álgebra lineal simplemente resolviendo el siguiente sistema de ecuaciones homogéneo:

$$
(A-I) \pi=0
$$

Las cadenas de Markov permiten tanto dar información a corto plazo (en nuestro caso cada 10 minutos) como analizar la evolución a largo plazo dando el estado predominante.

\section{METODOLOGÍA PARA EL DISEÑO DEL MODELO}

En este apartado tenemos como objetivo el mostrar la metodología usada para el diseño de un modelo para el análisis de la velocidad y dirección del viento mediante Cadenas de Markov lineales. Esta metodología se aplicará a un caso concreto con datos reales, en el apartado V.

El primer paso consiste en la recogida de datos y en la definición y asignación de los estados tanto para el caso de direcciones como el caso de velocidades del viento. Dicha lectura se realiza utilizando alguna herramienta de software como por ejemplo Matlab.

Una vez los datos han sido leídos y guardados el siguiente paso es categorizarlos para ello, se definen y asignan los estados. Se pueden definir tantos estados como sean necesarios, en función de la precisión y el rango del estudio deseados. En el caso de direcciones, se toma como referencia para la definición de estados la rosa de los vientos.

Como es bien sabido, ésta se puede dividir en distintos estados. Cuatro estados es el caso más sencillo, teniendo tan sólo las 4 direcciones principales (N, E, S, O). A partir de ahí, se obtiene más precisión aumentando el número de estados a $8,16,32$ y así sucesivamente. En cuanto a las velocidades, el número de estados puede variar de la manera que más convenga. La única referencia que se ha de tener en cuenta en el momento de definir los estados de velocidad es el motivo de estudio y el rango de velocidades deseadas para el mismo.

Se considera que la partición en 8 estados es adecuada para la realización de dicho estudio. Concretamente, se consideran los estados mostrados en las siguientes tablas.

TABLA I

ESTADOS ASIGNADOS A LAS DIRECCIONES

\begin{tabular}{|c|c|c|}
\hline Estado & Grados & Dirección \\
\hline 1 & $337,5<$ Valor $<=22,5$ & Norte \\
\hline 2 & $22,5<$ Valor $<=67,5$ & Noreste \\
\hline 3 & $67,5<$ Valor $<=112,5$ & Este \\
\hline 4 & $112,5<$ Valor $<=157,5$ & Sureste \\
\hline 5 & $157,5<$ Valor $<=202,5$ & Sur \\
\hline 6 & $202,5<$ Valor $<=247,5$ & Suroeste \\
\hline 7 & $247,5<$ Valor $<=292,5$ & Oeste \\
\hline 8 & $292,5<$ Valor $<=337,5$ & Noroeste \\
\hline
\end{tabular}

TABLA II

ESTADOS ASIGNADOS A LOS DATOS DE VELOCIDAD

\begin{tabular}{|c|c|c|}
\hline Estado & $\mathrm{m} / \mathrm{s}$ & $\mathrm{km} / \mathrm{h}$ (aprox) \\
\hline
\end{tabular}




\begin{tabular}{|c|c|c|}
1 & $0<$ Valor $<=0,0833$ & $0<$ Valor $<=0,3$ \\
\hline 2 & $0,0833<$ Valor $<=2,77$ & $0,3<$ Valor $<=10$ \\
\hline 3 & $2,77<$ Valor $<=5,55$ & $10<$ Valor $<=20$ \\
\hline 4 & $5,55<$ Valor $<=8,33$ & $20<$ Valor $<=30$ \\
\hline 5 & $8,33<$ Valor $<=11,11$ & $30<$ Valor $<=40$ \\
\hline 6 & $11,11<$ Valor $<=13,88$ & $40<$ Valor $<=50$ \\
\hline 7 & $13,88<$ Valor $<=16,66$ & $50<$ Valor $<=60$ \\
\hline 8 & $16,66<$ Valor & $60<$ Valor \\
\hline
\end{tabular}

El segundo paso es la generación de las matrices de transición de estados, estas matrices se construyen según la definición dada en el apartado anterior, teniendo en cuenta las frecuencias de cada estado contabilizadas a partir de la asignación de estados realizado en el primer paso. Estas matrices se utilizarán posteriormente, para el estudio de predicción de la dirección y velocidad del viento en un lugar previamente escogido. Obviamente, el tamaño de la matriz depende del número de estados que se han definido, en nuestro caso, las matrices son de tamaño $8 \times 8$.

$$
A=\left(\begin{array}{ccc}
p_{11} & \ldots & p_{18} \\
\vdots & & \vdots \\
p_{81} & \cdots & p_{88}
\end{array}\right)
$$

Una vez obtenidas las matrices de transición de estados se debe comprobar que dichas matrices cumplen las condiciones de cadena de Markov lineal.

Para ello, simplemente se debe comprobar que se cumple la siguiente igualdad relacionada con las propiedades de Markov:

$\operatorname{Pr}\left\{X_{2}=i_{2} \mid X_{1}=i_{1}\right\}=\operatorname{Pr}\left\{X_{2}=i_{2} \mid X_{0}=i_{0} ; X_{1}=i_{1}\right\}$

De esta forma, se compara la probabilidad de que un estado dado $X_{2}$, se dé después de que se haya dado otro estado $X_{1}$, con la probabilidad de que se dé este estado $X_{2}$ depués de que se haya dado el mismo estado $X_{1}$ a la vez que este se haya dado después de otro estado $\mathrm{X}_{0}$.

Obtenidos estos datos, simplemente se grafican tomando como eje de las abscisas las probabilidades primeras, y como ordenadas las probabilidades segundas, y se observa si la nube de puntos obtenida está alrededor de la bisectriz. En caso afirmativo, es decir si se obtiene una gráfica semejante a la de Fig. 1, entonces, se tiene que, los datos son modelables como cadenas de Markov.

(La gráfica de la Fig. 1 ha sido realizada con datos de una cadena de Markov auténtica en la que se observa como la nube de puntos está alrededor de la bisectriz).

A continuación y con las herramientas que nos brinda el álgebra lineal, se pasa a hacer el estudio analítico de las matrices estudiando los valores propios de dichas matrices ya que estos son los datos que nos permitirán valorar la dinámica de los datos a largo plazo.

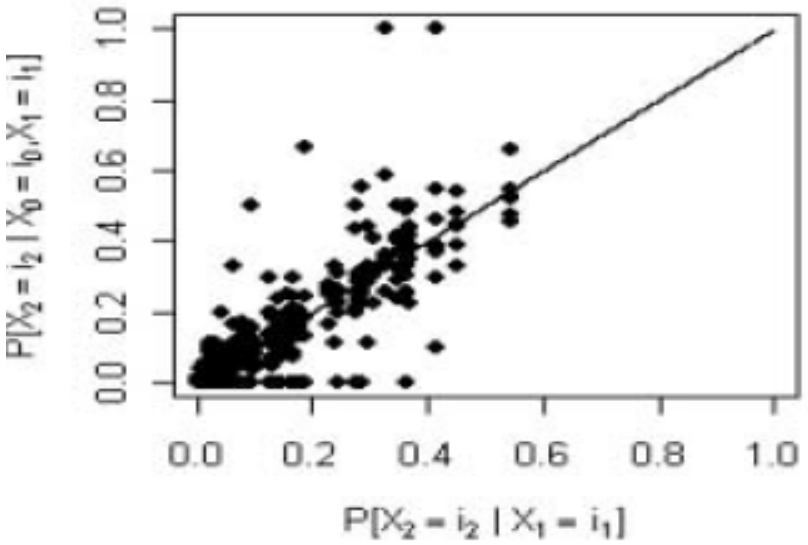

Figura 1. Gráfica de Propiedad de Markov, [4].

Se calculan los valores y vectores propios de las matrices necesarios para realizar el estudio de predicción de la velocidad y de la dirección del viento, cuyo resultado dependerá de los valores propios hallados. Si la matriz es positiva la matriz tiene un valor propio real y positivo simple y es mayor que el valor absoluto de cualquier otro valor propio de la matriz. Este valor propio tiene asociado un vector propio con todas las coordenadas positivas y se puede escoger de manera que todas sus coordenadas sumen 1. Este vector caracteriza el comportamiento a largo plazo de la cadena de Markov lineal, ya que nos informa de la probabilidad de que la cadena se encuentre en cada uno de los estados cuando ha transcurrido un número grande de unidades de tiempo.

El paso siguiente consiste en la búsqueda del punto de equilibrio estable, en caso de que ella exista, para concluir con el análisis de los resultados.

En el caso que nos ocupa, ambas matrices tienen el valor propio 1 como valor propio dominante y si las matrices son irreducibles, entonces existe distribución estacionaria para ambas. Y si además, las matrices $A$ son primitivas, entonces las distribuciones son independientes de los estados iniciales $\mathrm{p}(0)$.

De hecho en estas condiciones la distribución estacionaria es el vector propio de valor propio 1 normalizado, esto es el vector propio cuyas coordenadas suman 1 .

\section{CASO DE ESTUDIO}

A continuación aplicamos los resultados presentados a un caso concreto.

Se toma como fuente de información las direcciones y las velocidades del viento cuyas mediciones están expresadas en grados y en $\mathrm{m} / \mathrm{s}$ respectivamente, que han sido registradas por la estación meteorológica de Trinidad de Iturgoyen”, [7]. Dicha estación se halla ubicada en España en la comunidad autónoma de Navarra, a una altura de 1200 metros sobre el nivel del mar (m.s.n.m), con unas coordenadas de $\left(42^{\circ} 46^{\prime} 34^{\prime \prime} \mathrm{N}, 1^{\circ} 57^{\prime} 15^{\prime \prime} \mathrm{O}\right)$.

Los datos escogidos para el análisis son concretamente los que van desde el 23 de Marzo de 2015 hasta el 9 de Marzo de 2016, en intervalos de tiempo de 10 minutos. Recordemos que la dirección del viento se define como la orientación del 
vector del viento en la horizontal que para fines meteorológicos, se considera como la dirección desde la cual sopla el viento. La distribución de velocidades y de direcciones de viento en un emplazamiento se puede aproximar con precisión con una ley Weibull. Por ejemplo mostramos en la Fig. 2, el histograma de frecuencias acumuladas de velocidades de viento

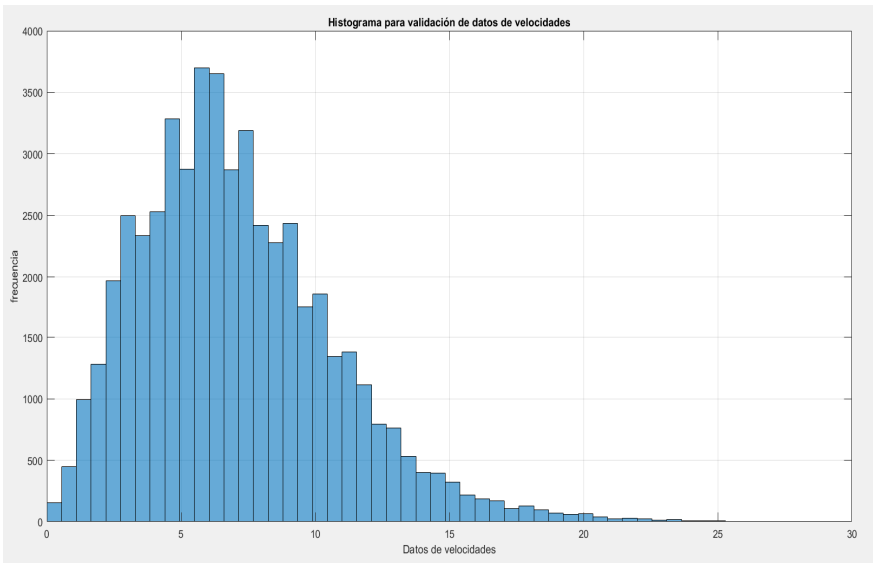

Figura 2. Histograma de frecuencias acumuladas de velocidades viento

El problema planteado para el caso de las velocidades es analizar las variaciones de las velocidades a lo largo del tiempo, en un rango de velocidades que incluye todas las velocidades posibles a las que un molino estaría en funcionamiento. La división de estados se hace desde $0 \mathrm{~m} / \mathrm{s}$ hasta valores superiores a $16,66 \mathrm{~m} / \mathrm{s}$ (que equivale a $60 \mathrm{~km} / \mathrm{h}$ ). Se definen 8 estados (los definidos en la tabla II) divididos de manera que el primero y el último son de referencia y los 6 del medio avanzan de $10 \mathrm{~km} / \mathrm{h}$. Por ejemplo, el estado 3 incluye las velocidades de $2,77 \mathrm{~m} / \mathrm{s}$ a $5,55 \mathrm{~m} / \mathrm{s}$, es decir de 10 a $20 \mathrm{~km} / \mathrm{h}$.

Para el caso de las direcciones el problema planteado es el de analizar las probabilidades de cambio de una dirección a otra del viento a partir de un muestra observada de direcciones del viento. Por ello, se han analizado estos datos con la finalidad de modelarlos como una cadena de Markov. Estos datos se han categorizado según los estados dados por la rosa de los vientos (los definidos en la tabla I).

El Análisis ha sido realizado siguiendo la metodología descrita y utilizando la herramienta Matlab para su desarrollo, se ha creado un programa con diversos ficheros cuyos algoritmos son aplicables a diferentes casos de estudio. De esta forma se consigue una herramienta de software aplicable para una amplia gama de estudios relacionados con la dirección y la velocidad del viento.

Aplicando pues, la metodología, empezamos recogiendo los datos y asignando los estados, después de la recogida de datos y la asignación de estados se construyen las matrices de transición de estados, que en este caso particular son las siguientes.

Matriz de transición de las direcciones tomadas:
( $\left.\begin{array}{lllllllll}0,9120 & 0,1142 & 0,0106 & 0,0020 & 0,0004 & 0,0022 & 0,0071 & 0,1534\end{array}\right)$ $\begin{array}{llllllllllll}0,0272 & 0,8421 & 0,0875 & 0,0037 & 0,0002 & 0,0006 & 0,0004 & 0,0014\end{array}$ $\begin{array}{lllllllllllllll}0,0004 & 0,0319 & 0,7148 & 0,0324 & 0,0010 & 0,0009 & 0,0004 & 0,0003\end{array}$ $\begin{array}{lllllllllllllll}0,0001 & 0,0017 & 0,1525 & 0,7408 & 0,0399 & 0,0034 & 0,0004 & 0,0001\end{array}$ $\begin{array}{llllllllllllllllll}0,0003 & 0,0017 & 0,0265 & 0,2104 & 0,9043 & 0,0964 & 0,0014 & 0,0001\end{array}$ $\begin{array}{lllllllllllll}0,0006 & 0,0032 & 0,0066 & 0,0087 & 0,0525 & 0,8246 & 0,0938 & 0,0038\end{array}$ $\begin{array}{lllllllllllllll}0,0009 & 0,0011 & 0,0013 & 0,0012 & 0,0007 & 0,0670 & 0,8340 & 0,0371\end{array}$

$\left.\begin{array}{llllllll}0,0581 & 0,0035 & 0 & 0,0004 & 0,0005 & 0,0045 & 0,0620 & 0,8035\end{array}\right)$

Matriz de transición de las velocidades tomadas:

$\left(\begin{array}{cccccccc}0,6666 & 0,0016 & 0 & 0 & 0 & 0 & 0 & 0 \\ 0,2916 & 0,8269 & 0,0583 & 0,0001 & 0 & 0 & 0 & 0 \\ 0 & 0,1699 & 0,8401 & 0,0907 & 0,0011 & 0 & 0 & 0 \\ 0,0416 & 0,0012 & 0,1007 & 0,8324 & 0,1235 & 0,0009 & 0,0006 & 0 \\ 0 & 0 & 0,0006 & 0,0761 & 0,8001 & 0,1738 & 0,0033 & 0,0012 \\ 0 & 0,0002 & 0 & 0,0003 & 0,0742 & 0,7447 & 0,2225 & 0,0037 \\ 0 & 0 & 0 & 0,0006 & 0,0009 & 0,0787 & 0,6742 & 0,1897 \\ 0 & 0 & 0 & 0 & 0 & 0,0016 & 0,0992 & 0,8052\end{array}\right)$

Observamos que ambas matrices son de tamaño $8 \times 8$, no negativas y han sido obtenidas a partir de las frecuencias de cada uno de los estados.

Siguiendo el algoritmo, el próximo paso consiste en estudiar el carácter markoviano de las matrices de transición y en calcular los vectores y valores propios de dichas matrices. También para estos cálculos se ha utilizado Matlab.

Con estas matrices tenemos la estimación puntual de los cambios de direcciones y velocidades del viento. Si se desea hacer inferencia sobre alguno de los datos se usarán niveles de plausibilidad o verosimilitud de 0.036 y 0.147 puesto que cumple teóricamente con una confianza asociada del $99 \%$ y $95 \%$ respectivamente.

Tal y como hemos indicado en el apartado IV.III, para probar el carácter markoviano de las matrices obtenidas, se ha de comparar las probabilidades que se obtienen de pasar de un estado a otro con las probabilidades de pasar de uno a otro teniendo en cuenta el estado anterior

Las gráficas correspondientes a dichos cálculos son las siguientes (Fig. 3 y Fig. 4).

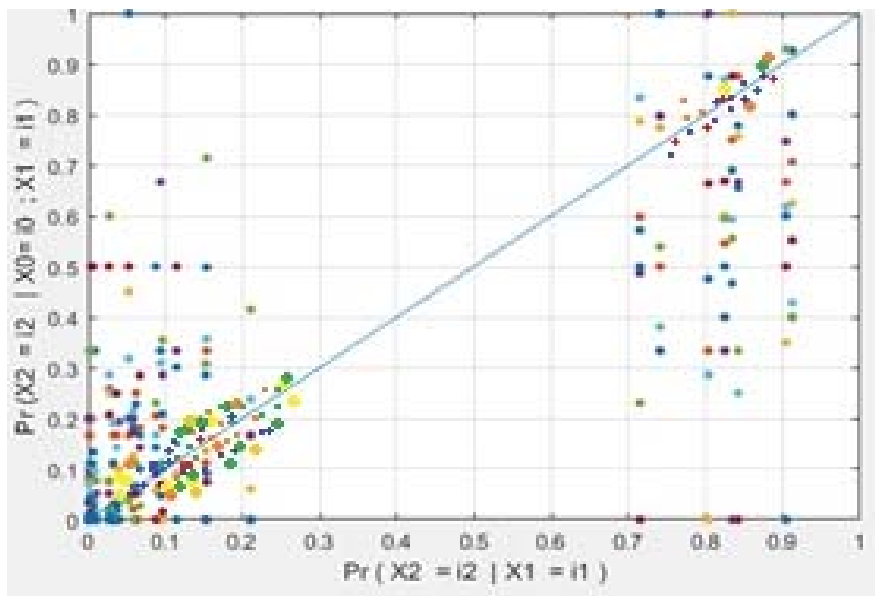

Figura 3. Comprobación carácter markoviano para las direcciones. 


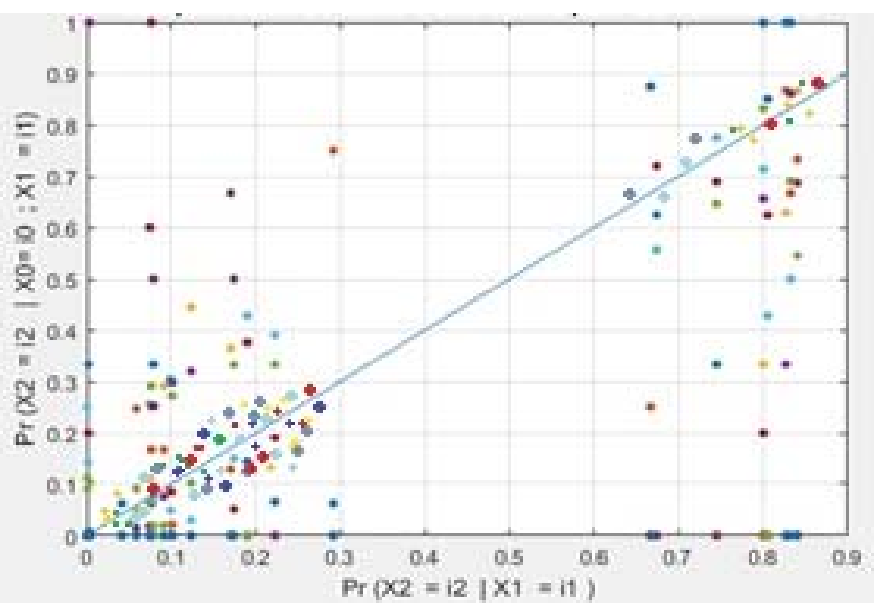

Figura 4. Comprobación carácter markoviano para las velocidades.

Hay que remarcar que los puntos de las gráficas próximos a la bisectriz son puntos compartidos por múltiples entradas, a diferencia de los puntos alejados que son puntos aislados, probando por tanto el carácter markoviano de las matrices de transición de estados.

Se muestran a continuación (Fig. 5 y Fig. 6), los datos tanto para las velocidades como para las direcciones de viento de un día al azar y se observa la regularidad de los datos, tanto para las velocidades como para las direcciones

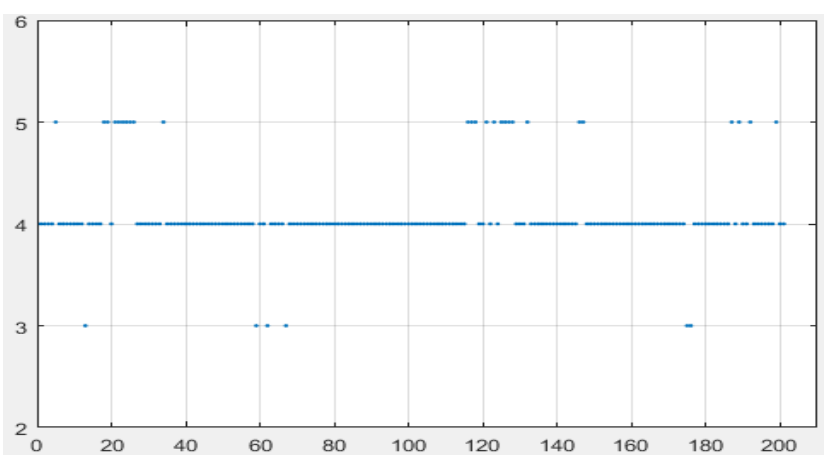

Figura 5. Datos para la velocidad de un día al azar.

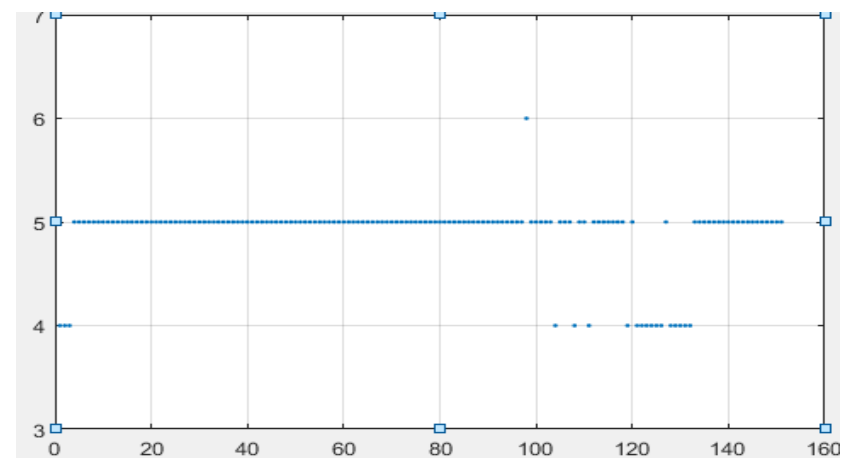

Figura 6. Datos para la dirección de un día al azar.

En estas gráficas se observa que tanto la velocidad como la dirección se mantienen prácticamente en el mismo estado durante todo el día.
Por otro lado estudiamos el espectro de las matrices, esto es el conjunto de valores propios de las matrices de transición. Los valores propios de la matriz de transición de estados para las velocidades son:

$0.514618063,0.653470593,0.6655826260 .7187930161$, 0.805422398, 0.947220023, 0.8854304,

Puesto que el valor propio dominante es la unidad y es simple, existe estado estacionario dado por el vector propio asociado al valor propio 1 . Dicho estado estacionario es el siguiente:

$(0.00047169, \quad 0.09482909,0.27764708,0.30889264$, $0.19059181,0.08206906,0.02966833,0.0158303)$.

Para el caso de las direcciones, los valores propios que se obtienen son:

$1, \quad 0.97766307, \quad 0.63569891, \quad 0.8957267, \quad 0.84188432$ $0.77788762,0.71597739,0.73163283$.

En dicho caso también, el valor propio dominante es la unidad y también es simple, por lo que existe estado estacionario que es el siguiente:

$(0.31353977, \quad 0.06576453, \quad 0.01483152, \quad 0.04729761$ $0.23417854,0.12116932,0.08029021,0.12292849)$.

Mostramos a continuación unas tablas conteniendo los resultados obtenidos sobre las distribuciones estacionarias tanto para las velocidades como para las direcciones.

TABLA III

TENDENCIA SOBRE VELOCIDADES

\begin{tabular}{|c|c|}
\hline Estado de Velocidad & $\mathbf{\%}$ \\
\hline 1 & 0,04716887 \\
\hline 2 & 9,48290873 \\
\hline 3 & 27,7647083 \\
\hline 4 & 30,8892644 \\
\hline & 19,0591805 \\
\hline 6 & 8,20690622 \\
\hline 7 & 2,96683287 \\
\hline 8 & 1,58303012 \\
\hline
\end{tabular}

TABLA IV TENDENCIA SOBRE DIRECCIONES

\begin{tabular}{|c|c|}
\hline Estado de Dirección & $\mathbf{\%}$ \\
\hline 1 & 31,3539771 \\
\hline 2 & 6,57645326 \\
\hline 3 & 1,48315199 \\
\hline 4 & 4,72976056 \\
\hline 5 & 23,4178544 \\
\hline
\end{tabular}




\begin{tabular}{|c|c|}
\hline 6 & 12,1169321 \\
\hline 7 & 8,02902144 \\
\hline 8 & 12,2928491 \\
\hline
\end{tabular}

Estas tendencias no dependen de las condiciones iniciales por lo que pasan a ser los vectores de máxima probabilidad.

Por tanto, se concluye que en la Estación meteorológica de Iturgoyen, la tendencia de las velocidades del viento oscila, en un $80 \%$ del tiempo aproximadamente entre los $10 \mathrm{~km} / \mathrm{h}$ y los $40 \mathrm{~km} / \mathrm{h}$. Además cabe destacar que prácticamente nunca (menos de un $10 \%$ del tiempo) el viento se encuentra en el estado 2 o 1 , lo que significaría una velocidad menor a $10 \mathrm{~km} / \mathrm{h}$ y por tanto insuficiente para la generación de energía eólica.

Por tanto, se puede decir que la estación de Iturgoyen, en lo que a las velocidades del viento se refiere, es un lugar muy bueno para colocar un parque eólico. Se recuerda que el rango de velocidades en las cuales se genera más energía eólica va desde los $7 \mathrm{~m} / \mathrm{s}$ hasta los $18 \mathrm{~m} / \mathrm{s}$, es decir desde los 25 $\mathrm{km} / \mathrm{h}$ hasta los $65 \mathrm{~km} / \mathrm{h}$ aproximadamente. Como se puede ver en la distribución estacionaria, a lo largo de todo el año, la tendencia es que las velocidades superen $10520 \mathrm{~km} / \mathrm{h}$ en un $62,7 \%$.

Con respecto a las direcciones del viento la dirección más probable a lo largo del tiempo es la del estado 1, es decir el Norte, con un $31,35 \%$ de probabilidad. Sin embargo, también hay una tendencia hacia el Sur, estado 5, es del $23,42 \%$. Esto evidencia una cierta inestabilidad en la dirección del viento aunque como se ha observado en la gráfica de un día al azar no son cambios continuos de viento que harían incontrolable el posicionamiento del eje del aerogenerador, en caso de no disponer de sistema de control de la dirección. En el caso de disponer de dicho controlador, el conocimiento de la variación de las direcciones permite hacer un buen diseño e implementación del sistema de control, optimizando así el rendimiento del aerogenerador.

\section{CONCLUSIONES}

El primer paso para la posible instalación de un parque eólico, en un lugar concreto es el de hacer un estudio de las direcciones y velocidades de los vientos de la zona donde se vayan a colocar los aerogeneradores.

Los autores enfatizan sobre el uso de las cadenas de Markov como herramienta matemática que permite determinar el acontecimiento de un suceso futuro dependiente únicamente de un suceso inmediatamente anterior. Además tiene la propiedad de permitir hallar la probabilidad de que un sistema se encuentre en un cierto estado en un momento dado y predecir el comportamiento del sistema a través del tiempo.

Si bien la teoría sobre cadenas de Markov salió a la luz a principios del siglo XX es una técnica innovadora, de gran utilidad y rigor científico que sirve tanto modelar el comportamiento de ciertos sistemas tanto de ingeniería, economía como de medicina entre otros, bajo condiciones de incertidumbre a través del tiempo.

La metodología presentada basada en el álgebra lineal, permite analizar la tendencia de las velocidades y de las direcciones del viento. La descripción matricial del proceso permite la utilización del álgebra lineal, por lo que el estudio de la evolución del sistema se traduce en el cálculo de la potencia de una matriz para lo que es fundamental la obtención de los valores propios de cada uno de los modelos.

De entre el conjunto de valores propios el dominante permite estudiar la tendencia del sistema. El conocimiento de las direcciones y velocidades predominantes del viento permite discernir sobre la orientación de los aerogeneradores en vistas a la producción de la mayor cantidad de energía posible.

Dicha metodología se ha aplicado al caso concreto de la estación meteorológica situada en Iturgoyen. El hecho de obtener estados estacionarios en los modelos diseñados para el estudio permite obtener vectores de máxima probabilidad permitiendo por tanto diseñar controladores para los aerogeneradores que permitan optimizar el rendimiento de los parques eólicos.

\section{AGRADECIMENTOS}

Los autores quieren agradecer las conversaciones con José Luis Domínguez-García investigador del Institut de recerca en Energia de Catalunya (IREC), cuyos comentarios han mejorado sustancialmente el trabajo realizado. También queremos agradecer muy profundamente a los revisores por sus valiosos comentarios que han dado al artículo la calidad que tiene.

\section{REFERENCIAS}

[1] R.B. Bapat, T.E.S. Raghavan, Nonnegative Matrices and Applications, Encyclopedia of Mathematics and its Applications, no 64, Cambridge University Press, 1997.

[2] M.W. Carter. C.C. Price. Operations Research: A practical introduction. CRC Press. USA. 2001.

[3] J.L. Domínguez García, M.I. García Planas. Introducción a la teoría de matrices positivas. Aplicaciones. Iniciativa Digital Politècnica, 2013.

[4] M.C. Palafox Duarte. Inferencia Estadística para Cadenas de Markov. Tesis doctoral, México, 2009.

[5] F.A. Sonnenberg, J.R. Beck. Markov models in medical decision making. Medical Decision Making, vol. 13, pp. 322-338, 1993.

[6] D.J. White. Further real applications of Markov decision processes. Interfaces, Volumen. 18. Número 5, pp. 55-61. 1988.

[7] Meteorología y climatología de Navarra, URL: http:// meteo.navarra.es/estaciones/estacion.cfm?IDEstacion $=29$

[8] E. Seneta. Non-negative Matrices and Markov Chains. Springer Science \& Business Media, 2006

[9] Y. Nesterova, A. Nemirovskib, Finding the stationary states of Markov chains by iterative methods. Applied Mathematics and Computation, vol. 255, pp. 58-65, 2015.

[10] J. Mur Amada. Wind power variability in the grid. PhD Thesis Zaragoza 2009.

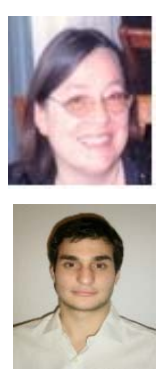

María Isabel García-Planas received the $\mathrm{PhD}$ in Mathematics from Universitat Politècnica de Catalunya, Barcelona Spain, in 1995. Her work had been centered on Linear Algebra, Systems and Control Theory. She has authored over eighty papers and serves on the referee on several journals.

Tornike Gongadze has recently obtained his degree in Industrial Technology Engineering presenting a bachelors thesis titled 'Design and validation of a predictive model for wind direction by means of Markov chains'. He will perform his MSc programme in the University of Twente, Netherlands. 